\title{
Progonóstico visual para facectomia: Retinômetro de Heine e orifício estenopéico múltiplo
}

\author{
Visual prognosis for lens extraction: Heine retinometer and multiple pinhole
}

\author{
Frederico Augusto Costa Reis ${ }^{1}$ \\ Ralph Cohen ${ }^{2}$ \\ Carlos Roberto Neufeld ${ }^{3}$ \\ Ana Carolina Toledo Dias ${ }^{4}$ \\ Daniel de Souza Pereira ${ }^{1}$
}

\section{RESUMO}

Objetivo: Avaliar a acurácia do retinômetro de Heine e do orifício estenopéico múltiplo no prognóstico da acuidade visual pós-facectomia. Métodos: Foram examinados 65 olhos com o retinômetro de Heine e com o orifício estenopéico múltiplo. Após a facectomia os pacientes foram submetidos à refração e os resultados comparados com a previsão dos instrumentos. O grupo 1 é formado por pacientes com acuidade visual pior que 20/100 e o grupo 2 melhor ou igual a 20/100. Resultado: O retinômetro de Heine teve bons resultados em $21 \%$ e $44 \%$, nos grupos 1 e 2 respectivamente. O orifício estenopéico múltiplo teve bons resultados em $26 \%$ e $52 \%$, nos grupos 1 e 2 respectivamente. Conclusão: O retinômetro de Heine tem acurácia semelhante ao orifício estenopéico múltiplo no prognóstico da acuidade visual pós-facectomia. Os instrumentos não devem ser usados para contra-indicar a facectomia devido ao grande número de resultados falso-negativos.

Descritores: Catarata; Extração de catarata; Acuidade visual; Testes visuais; Percepção visual; Facoemulsificação; Interferometria
Trabalho realizado na Santa Casa de Misericórdia de São Paulo.

${ }^{1}$ Fellow da seção de Retina e Vítreo, do Departamento de Oftalmologia da Santa Casa de Misericórdia de São Paulo.

${ }^{2}$ Doutor em Oftalmologia, chefe da seção de Glaucoma do Departamento de Oftalmologia da Santa Casa de Misericórdia de São Paulo.

3 Assistente da seção de Uveíte, do Departamento de Oftalmologia da Santa Casa de Misericórdia de São Paulo.

${ }^{4}$ Fellow da seção de Óculo-plástica, do Departamento de Oftalmologia da Santa Casa de Misericórdia de São Paulo.

Endereço para correspondência: Rua Marquês de Itú, 545, Apt 81, CEP 01223-001 - São Paulo (SP)

E-mail: fredacreis@uol.com.br

Nota Editorial: Pela análise deste trabalho e por sua anuência na divulgação desta nota, agradecemos ao Dr. Breno Barth.

Recebido para publicação em 14.08.2003

Versão revisada recebida em 12.04.2004

Aprovação em 04.06.2004

\section{INTRODUÇ̃̃̃O}

A crescente exigência do paciente, aliada ao interesse do médico em avaliar qual o benefício visual após a facectomia fizeram com que nas últimas décadas vários instrumentos fossem testados com o objetivo de auxiliar o médico a predizer a acuidade visual (AV) do paciente, após a facectomia $^{(1)}$.

O potential acuity meter (PAM) prevê em $73 \%$ dos casos a AV dos pacientes $^{(1)}$; a acurácia do interferômetro a laser é de $88 \%{ }^{(2)}$ e a sensibilidade e a especificidade do "oscillatory displacement threshold" é de $100 \%$ e $98 \%$ respectivamente ${ }^{(3)}$.

Atualmente, os instrumentos mais estudados para predizer a AV pósfacectomia são o PAM e a interferometria a laser. Embora diversos trabalhos já tenham comprovado a sua confiabilidade nos pacientes com AV melhor que 20/100, o alto custo continua sendo um problema ${ }^{(4-6)}$. Uma alternativa seria o uso em larga escala do retinômetro de Heine( $\mathrm{RH})$ e/ou do orifício estenopéico múltiplo (OEM), devido ao fácil manuseio e custos bem mais baixos, em comparação com os outros instrumentos. No entanto, existem poucos trabalhos científicos sobre o uso do RH e do OEM para prognóstico da AV central pós-facectomia, restringindo, dessa maneira, a utilização desses instrumentos ${ }^{(7-8)}$.

O OEM é utilizado rotineiramente para mensuração da $\mathrm{AV}$ à distância em pacientes com ametropias, sem a necessidade de correção óptica; este 
recurso melhora a $\mathrm{AV}$ por aumentar a profundidade de foco e condensar a imagem projetada na fóvea; o tamanho ideal do orifício é de $0,94 \mathrm{~mm}$ a $1,75 \mathrm{~mm}$, valores maiores diminuem a profundidade do foco e os menores diminuem a resolução visual por difração ${ }^{(9)}$.

O RH é um aparelho manual, com formato semelhante ao oftalmoscópio direto, que projeta linhas paralelas com diferentes freqüências espaciais na mácula, através de uma área do cristalino sem opacidade. Essas linhas podem ser verticais, horizontais ou inclinadas, com alto contraste e bordas bem $\operatorname{definidas}^{(7)}$.

Este trabalho teve como objetivo avaliar comparativamente a acurácia individual do RH e do OEM no prognóstico da AV pós-facectomia, determinando a sensibilidade e especificidade dos aparelhos.

\section{MÉTODOS}

Foram avaliados prospectivamente, de forma aleatória, 65 olhos de 54 pacientes ( 28 homens e 26 mulheres), com idade média de $78 \pm 10,6$ anos, que iriam ser submetidos à facectomia, tanto pela técnica extra-capsular manual, como por facoemulsificação, na Clínica Oftalmológica da Santa Casa de Misericórdia de São Paulo, entre junho de 2000 e junho de 2001.

Todos os pacientes obedeceram aos critérios de inclusão e exclusão e foram informados sobre os procedimentos aos quais iriam submeter-se.

Toda a coleta de dados foi realizada pelo autor.

\section{Critérios de inclusão}

1) Portadores de catarata senil;

2) Acuidade visual pior ou igual a 20/40 com a melhor correção óptica para longe, no olho a ser operado.

\section{Critérios de exclusão}

1) Lesões do segmento anterior que impedissem a transmissão de luz ao pólo posterior;

2) Complicações cirúrgicas ou pós-cirúrgicas, que pudessem prejudicar a AV final;

3) Pacientes submetidos a capsulotomia posterior menos de 60 dias antes da refração pós-operatória.

\section{Protocolo da avaliação}

Foi mensurada a acuidade visual dos pacientes com melhor correção óptica para longe, com a tabela de Snellen graduada para cinco metros. Posteriormente, todos tiveram suas pupilas dilatadas com uma gota de tropicamida a $1 \%$ e de fenilefrina a $10 \%$, nesta ordem, com intervalo entre as instilações de cinco minutos. Os testes foram realizados 20 minutos após a instilação do último colírio.

Apenas dois pacientes não foram submetidos ao RH e um ao OEM, por não entenderem o exame. Os instrumentos utilizados foram o RH (Heine instruments, Germany) e o OEM, com orifícios de $1 \mathrm{~mm}$ de diâmetro.
O RH e o OEM foram utilizados sempre na mesma sala com a mesma iluminação. Para mensurar a acuidade visual nos pacientes submetidos ao OEM, foi usada a tabela de Rosenbaum, a uma distância de $35 \mathrm{~cm}$ do olho do paciente, iluminada pela luz branca do oftalmoscópio Welch Allyn de 3,5 volts. Não foi usada correção óptica para perto, já que o orifício estenopéico dispensa a correção.

Os pacientes selecionados para a segunda fase do trabalho foram reexaminados a partir do segundo mês após a cirurgia, com mensuração da AV com melhor correção óptica para longe, na mesma sala em que foram realizados os testes précirúrgicos.

Os pacientes foram divididos em dois grupos, de acordo com a melhor AV pré-operatória. O primeiro grupo (1) foi formado por pacientes com acuidade visual pior que 20/100 e o segundo grupo (2) por pacientes com acuidade visual melhor ou igual a $20 / 100$.

O resultado foi considerado bom quando a AV pós-operatória com a melhor correção óptica foi igual à prevista ou se a diferença foi de \pm 1 linha da tabela de Snellen. Foi considerado ruim quando a diferença foi maior que \pm 1 linha.

\section{RESULTADOS}

Os resultados encontrados com o RH e o OEM são descritos nas tabelas e gráficos abaixo.

$\mathrm{Na}$ análise dos resultados do $\mathrm{RH}$, foi comprovado que apenas o valor do teste pré-operatório influenciou a acurácia do exame, $p=0,0009$ ( $t$-Student), quanto maior a AV prevista pelo aparelho mais confiável o resultado. A AV pré-operatória não demonstrou ser fator prognóstico apesar do valor de $p$ ter sido limítrofe, 0,052 (qui-quadrado).

Com relação ao OEM, a análise estatística comprovou que tanto a AV pré-operatória quanto o próprio valor do teste foram variáveis que influenciaram a acurácia dos resultados (quanto maior a AV do paciente e a prevista pelo instrumento mais confiável o resultado), sendo estatisticamente significantes, com $p=0,0065$ ( $t$-Student) e $p=0,0002$ (soma de pontos de Wilcoxon) respectivamente; os pacientes com AV pior que 20/100 tiveram pior resultado.

\begin{tabular}{|c|c|c|c|c|}
\hline & \multicolumn{2}{|c|}{$\begin{array}{l}\text { Retinômetro } \\
\text { de Heine }\end{array}$} & \multicolumn{2}{|c|}{$\begin{array}{c}\text { Orifício estenopéico } \\
\text { múltiplo }\end{array}$} \\
\hline & Bom & Ruim & Bom & Ruim \\
\hline$A V<20 / 100$ & $8(21 \%)$ & 30 (79\%) & $10(26 \%)$ & $29(74 \%)$ \\
\hline$A V \geq 20 / 100$ & 11 (44\%) & $14(56 \%)$ & $13(52 \%)$ & $12(48 \%)$ \\
\hline Total & 19 (30\%) & 44 (70\%) & $23(36 \%)$ & $41(64 \%)$ \\
\hline
\end{tabular}




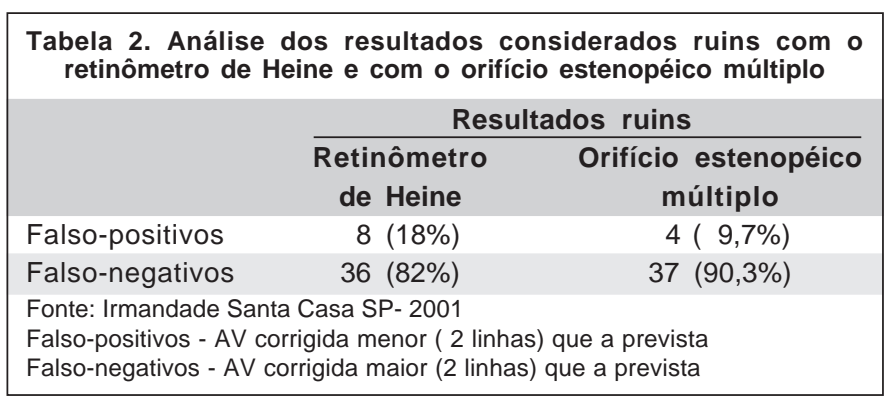

\section{DISCUSSÃO}

Os resultados considerados como falso-positivos foram os de AV pós-operatória menores que os previstos pelos instrumentos e nesse trabalho representaram um número pequeno dos resultados ruins. A maioria dos pacientes com este tipo de resultado apresentava alteração macular $(75 \%$ no RH e no $\mathrm{OEM}$ ), observação semelhante à descrita na literatura ${ }^{(2)}$. Os pacientes em que ocorreram falsos positivos foram os mesmos tanto com OEM como com RH, demonstrando concordância de $100 \%$.

A grande quantidade de resultados ruins com $\mathrm{RH}$, sendo a maioria falso-negativos, foi maior que a encontrada na literatura $(70 \% \times 32 \%)^{(7)}$. Entretanto no trabalho publicado, não houve descrição da amostra ou da avaliação dos resultados, impossibilitando a comparação com o presente estudo. Talvez essa diferença se deva à média das $\mathrm{AV}$ deles, maior que a da presente pesquisa ou por considerarem naquele estudo, uma margem de AV mais ampla, propiciando menor número de resultados ruins. Quando o RH é comparado com o PAM, já que ambos têm mesmo mecanismo de ação, o resultado é similar a alguns trabalhos da literatura ${ }^{(4-9)}$. Nos trabalhos em que o PAM foi considerado melhor, os autores consideraram como bom prognóstico uma faixa ampla de AV pós-operatória, fazendo com que o número de bons resultados aumentasse ${ }^{(4,10)}$.

Com relação ao OEM, o número dos bons resultados encontrados foi semelhante ao encontrado na literatura ${ }^{(8)}$, $(26 \%$ x $28 \%)$ no grupo 1 , porém foi melhor $(52 \%$ x $42 \%)$ no grupo 2 .

A maioria dos pacientes nesse trabalho apresentava opacidades corticais intensas ou catarata subcapsular posterior que comprovadamente impedem a projeção de imagens na retina ${ }^{(8)}$, fazendo com que o número de resultados ruins aumentassem. O edema cistóide de mácula significante, que aparece entre 4 e 12 semanas após a cirurgia, em $20 \%$ dos pacientes submetidos a facectomia extracapsular ${ }^{(11)}$, pode contribuir para os resultados falso-positivos. Nossos pacientes foram submetidos ao exame fundoscópico após a cirurgia e não foi detectado edema cistóide significativo em nenhum paciente na fase da avaliação ( $\geq 2$ meses pós-cirurgia), porém não se realizou angiofluoresceinografia.

A interpretação dos gráficos 1 e 2 construídos a partir da análise de regressão logística demonstra qual a $\mathrm{AV}$ em que os

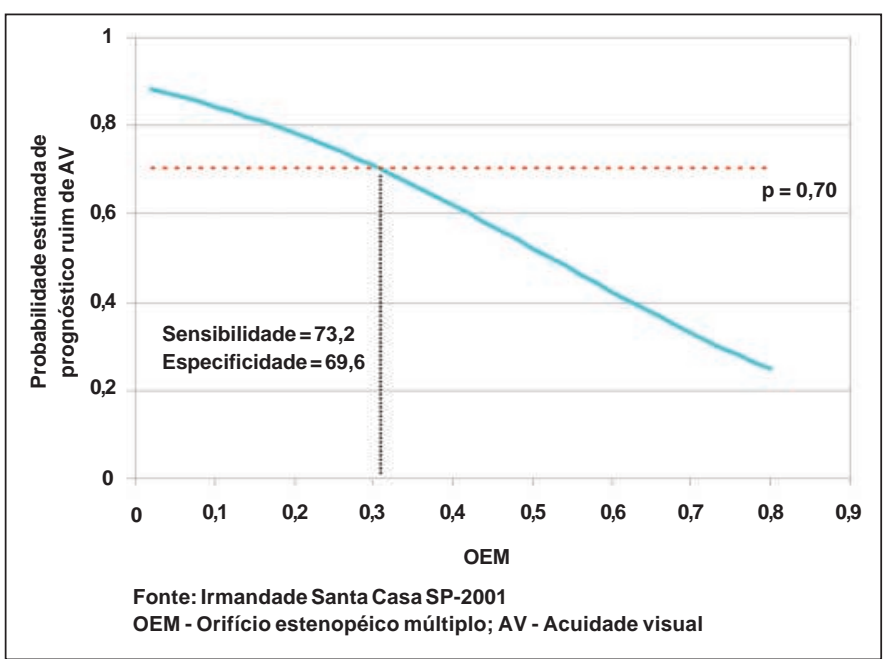

Gráfico 1 - Sensibilidade e especificidade do orifício estenopéico múltiplo por análise de regressão logística

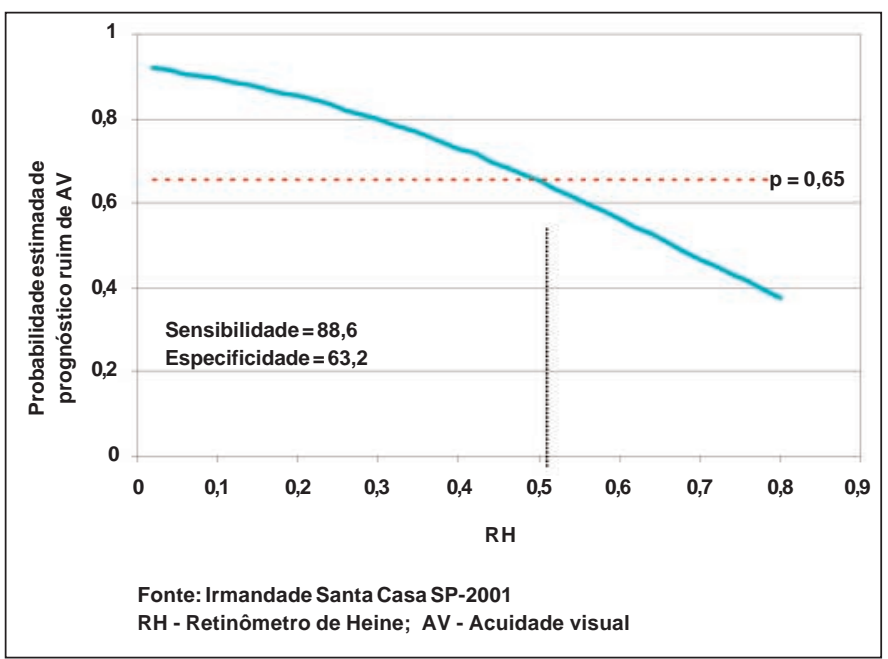

Gráfico 2 - Sensibilidade e especificidade do retinômetro de Heine por análise de regressão logística

aparelhos aliam maior sensibilidade a maior especificidade, que foi de 20/40 para o RH e 20/60 para o OEM. Com esses dados, é necessário fazer-se novo trabalho, com novos pontos de corte de acuidade visual, para se determinar a acurácia dos aparelhos.

Os dois testes tiveram resultados ruins nos pacientes em que a propedêutica pré-operatória foi mais difícil, pela opacidade do cristalino. Nos outros, apenas com a história pregressa e a avaliação oftalmológica completa, na maioria das vezes se conseguiu predizer com boa acurácia a AV pós-operatória, afirmação teórica comprovada por outro estudo ${ }^{(5)}$.

Se as melhores previsões ocorreram nos pacientes em que era mais fácil a avaliação macular, apenas pelo exame físico, talvez o uso desses instrumentos adjuvantes não tenha função tão importante e por isso deva-se preferir a utilização de aparelhos com menor custo. 


\section{CONCLUSÕES}

1) O retinômetro de Heine tem acurácia semelhante ao orifício estenopéico múltiplo no prognóstico da AV pós-operatória dos pacientes submetidos a facectomia.

2) Os dois testes não devem ser usados como único parâmetro para se contra-indicar a facectomia, pelo grande número de falso-negativos, principalmente nos pacientes com AV pré-operatória menor que 20/100.

3) O OEM proporciona menor número de resultados falsopositivos, que o RH.

4) Pacientes com alterações maculares têm maior proporção de falso-positivos.

\section{ABSTRACT}

Purpose: To evaluate Heine retinometer and multiple pinhole accuracy in the prognosis of the visual acuity after lens extraction. Methods: 65 eyes were examined with Heine retinometer and with multiple pinhole. After surgery the patients were submitted to refraction and the result were compared with the prediction by the instruments. Group 1 is formed of patients with visual acuity worse than $20 / 100$ and group 2, better or equal to 20/100. Results: The Heine retinometer obtained good results in $21 \%$ and $44 \%$ of patients in both groups ( 1 and 2 ) respectively. The multiple pinhole had good results in $26 \%$ and $52 \%$ of patients concerning both groups (1 and 2) respectively. Conclusion: The Heine retinometer has an accuracy similar to the multiple pinhole in the prognosis of visual acuity after lens extraction. The instruments should not be used to contraindicate lens extraction due to the large number of false negative results.

Keywords: Cataract; Cataract extraction; Visual acuity; Visual tests; Visual perception; Phacoemulsification

\section{REFERÊNCIAS}

1. Smiddy WE, Radulovic D, Yeo JH, Stark WJ, Maumenee E. Potential acuity meter for predicting visual acuity after Nd: YAG posterior capsulotomy. Ophthalmology. 1986; 93(3):397-400.

2. Datiles MB, Edwards PA, Kaiser-Kupfer MI, McCain L, Podgor M. A comparative study between the PAM and laser interferometer in cataracts. Graefe's Arch Clin Exp Ophthalmol. 1987;255(6):457-60.

3. Barrett BT, Davison PA, Eustace P. Clinical comparison of three techniques for evaluating visual function behind cataract. Eye. 1995;9(Pt 6):722-7.

4. Severin TD, Severin SL. A clinical evaluation of the potential acuity meter in 210 cases. Ann Ophthalmol. 1988;20(10):373-5.

5. Miller ST, Graney MJ, Elan JT, Applegate WB, Freeman JM. Predictions of outcomes from cataract surgery in elderly persons. Ophthalmology. 1988, 95(8):1125- 9.

6. Lasa MS, Datiles MB, Freidlin V. Potential vision tests in patients with cataracts Ophthalmology. 1995;102(7):1007-11.

7. Tharp A, Cantor L, Yung CW, Shoemaker J. Prospective comparision of the Heine Retinometer with the Mentor Guyton-Minkowski potential acuity meter for the assessment of potential visual acuity before cataract surgery. Ophthalmic Surg. 1994;25(9):576-9. Comment In: Ophthalmic Surg. 1994;25(9):657.

8. Melki AS, Safar A, Martin J, Ivanova A, Adi M. Potencial acuity pinhole. A simple method to measure potential visual acuity in patients with cataracts, comparison to potential acuity meter. Ophthalmology. 1999;106(7):1262-7. Comment In: Ophthalmology. 2000;107(4):623-4.

9. Lebensohn JE. The pinhole test (editorial). Am J Ophthalmol. 1950,33:1612-4.

10. Fish GE, Birch DG, Fuller DG, Straach R. A comparison of visual function tests in eyes with maculopathy. Ophthalmology. 1986;93(9):1177-82.

11. Ahmed I, Ai E. Cystoid macular edema. In: Yanoff M, Duker JS, editors. Ophthalmology. London: Mosby; 1999. p.1-6.

\section{CONGRESSO DA SOCIEDADE DE OFTALMOLOGIA DO TRIÂNGULO MINEIRO}

\section{8 c 20 de novembro de 2004 UBERLANDIA - MC}

\section{INFORMAÇÕES:}

Tel./Fax: (34) 3219-3333

E-mail: interld@netsite.com.br 